\title{
A Traditional Chinese Medicine Approach in Treating Depression by Promoting Liver Qi Circulation: A Western Medicine Perspective
}

\author{
Raphaella Wai Lam So, Hoi Shan Wong, Kam Ming Ko* \\ Division of Life Science, Hong Kong University of Science \& Technology, Hong Kong, China \\ Email: *bcrko@ust.hk
}

Received 22 August 2015; accepted 24 November 2015; published 27 November 2015

Copyright (C) 2015 by authors and Scientific Research Publishing Inc.

This work is licensed under the Creative Commons Attribution International License (CC BY). http://creativecommons.org/licenses/by/4.0/

cC) (7) Open Access

\begin{abstract}
Depression is a prevalent mental illness in developed countries. In Western medicine, experimental and clinical investigations have demonstrated that depression is associated with the dysregulation of neurotransmitter signaling, and symptoms of depression can be alleviated by therapeutic intervention. However, patients taking antidepressant drugs often experience serious side effects and high relapse rates. On the other hand, traditional Chinese medicine (TCM) views depression as a manifestation of liver qi stagnation. Practitioners of TCM have long been treating depression with herbs that promote qi circulation in the liver. In this article, we offer a hypothesis stating the biochemical basis of the linkage between liver qi stagnation and depression. Liver qi is involved in the processing of macronutrients into molecules to fuel energy metabolism in brain neurons, as well as the synthesis of plasma proteins that maintain blood circulation to the brain, thereby enabling these fuel molecules to be delivered to the brain. In cases of liver qi stagnation, the failure in delivering sufficient fuel molecules to the brain disrupts mitochondrial ATP production in neurons. Because neurotransmitter release and neurotropin transport are driven by ATP, the deficiency in release and transport processes resulting from insufficient ATP production could lead to depression. Therefore, if liver qi stagnation is causally related to the pathogenesis of depression, the promotion of liver qi circulation by Chinese herbs might offer a promising prospect for the effective treatment of depression.
\end{abstract}

\section{Keywords}

Mitochondrial ATP, Liver “Qi” Stagnation, Depression, Traditional Chinese Medicine

\footnotetext{
${ }^{*}$ Corresponding author.
} 


\section{Introduction}

Depression is a common disorder affecting over 350 million people worldwide, particularly in industrialized countries, making it the leading cause of disability [1]. In Hong Kong, the twelve-month prevalence of major depressive disorders is $8.4 \%$ [2]. Depressed patients exhibit a wide range of affective, cognitive, behavioral and physiological symptoms. They experience sadness, anxiety, feelings of worthlessness and guilt; they engage in self-denigration and rumination; they experience anhedonia, tearfulness, psychological retardation and undefined pain; they stop practicing personal hygiene and they exhibit changes in appetite, sleep, and libido [3] [4]. In the worst-case scenario, depression can even lead to suicide [1].

\section{Pathogenesis of Depression in Western Medicine}

Western medicine describes the etiology of depression in terms of biological, psychological, social and sociocultural factors. Biologists attribute depressive symptoms to genetic factors, disturbances in circadian rhythm, high cortisol levels, defective negative feedback in the hypothalamic-pituitary-adrenal (HPA) axis, and abnormalities in neurotransmission and brain structure. Specifically, it is believed that deficient serotonergic signaling has pathological implications in depression [5]-[7].

When an individual perceives threat, he/she elicits a stress (fight-or-flight) response involving the amygdala, which is the center of emotion in the brain. The amygdala signals the hypothalamus to secrete corticotropin-releasing factor (CRF). CRF then stimulates the release of adrenocorticotropic hormone (ACTH) from the anterior pituitary gland. ACTH is carried through the bloodstream to the adrenal cortex, where it enhances synthesis and release of glucocorticoids such as cortisol [7]. Thus, hypercortisolemia and stress are causally linked by the HPA axis. Chronic stress, therefore, repeatedly exposes an individual to increased levels of cortisol. Hypercortisolemia promotes the release of pro-inflammatory cytokines, causing neuroinflammation. Both glucocorticoids and pro-inflammatory cytokines inhibit the synthesis of serotonin by diverting its precursor, tryptophan, down the tryptophan-kynurenine pathway. Moreover, glucocorticoids also bind to mineralocorticoid receptors, which inhibit the function of serotonin receptors. In this regard, the so-called "inflammation hypothesis" relates chronic stress and hypercortisolemia to depression, wherein hypercortisolemia affects the normal synthesis and function of serotonin. In addition, activation of the tryptophan-kynurenine pathway produces the neurotoxin quinolinic acid, which activates the glutamatergic pathway and leads to neurodegeneration in the corticolimbic system, including the hippocampus and prefrontal cortex in the brain [8].

Under normal conditions, high cortisol levels activate glucocorticoid receptors in the cytoplasm, thereby producing negative feedback within the HPA axis and inhibiting further release of CRF and ACTH [7]. However, the chronic stress-induced neuroinflammation undermines this feedback mechanism by inducing glucocorticoid resistance and by further activating the HPA axis directly. First, pro-inflammatory cytokines inhibit the function of glucocorticoid receptors by favoring isoforms that are less effective at binding ligands, disrupting their translocation from the cytoplasm to the nucleus and affecting the subsequent gene transcription. This causes dysfunctional negative feedback of the HPA axis. Second, pro-inflammatory cytokines can also stimulate the HPA axis directly, but the detailed mechanism has yet to be elucidated [9]. Therefore, by inducing neuroinflammation, chronic stress not only abolishes the negative feedback control of the HPA axis, but it also induces a feed-forward cycle of hypercortisolemia through direct cytokine action. In this connection, Figure 1 depicts various pathways that can contribute to the development of depression. Chronic stress disrupts the negative feedback response in the HPA axis, down-regulates GABAergic signaling, and up-regulates glutamatergic signaling. Downstream effects of these processes include the suppression of neurogenesis and the induction of apoptosis in the corticolimbic system, with a resulting decrease in serotonergic signaling, thereby giving rise to depression.

Abnormalities in neurotransmission can also contribute to depression. After a chemical signal is transduced, the actions of neurotransmitter molecules are terminated by neuronal reuptake and/or degradation. Increased neurotransmitter degradation and/or reuptake can result in excessively low levels of serotonin, norepinephrine and dopamine in synaptic clefts, resulting in defective transmission of neural signals. It is worth noting here that pro-inflammatory cytokines have also been shown to promote reuptake of serotonin through activating its transporters, thereby decreasing the concentration of serotonin at the synapse where it functions as a neurotransmitter [8].

In fact, altered neurotransmission has been demonstrated to be biochemical basis for antidepressant drug action in Western medicine. As their names suggest, selective serotonin reuptake inhibitors (SSRIs) and serotonin-norepinephrine reuptake inhibitors (SNRIs) block the reuptake of these neurotransmitters into presynaptic 


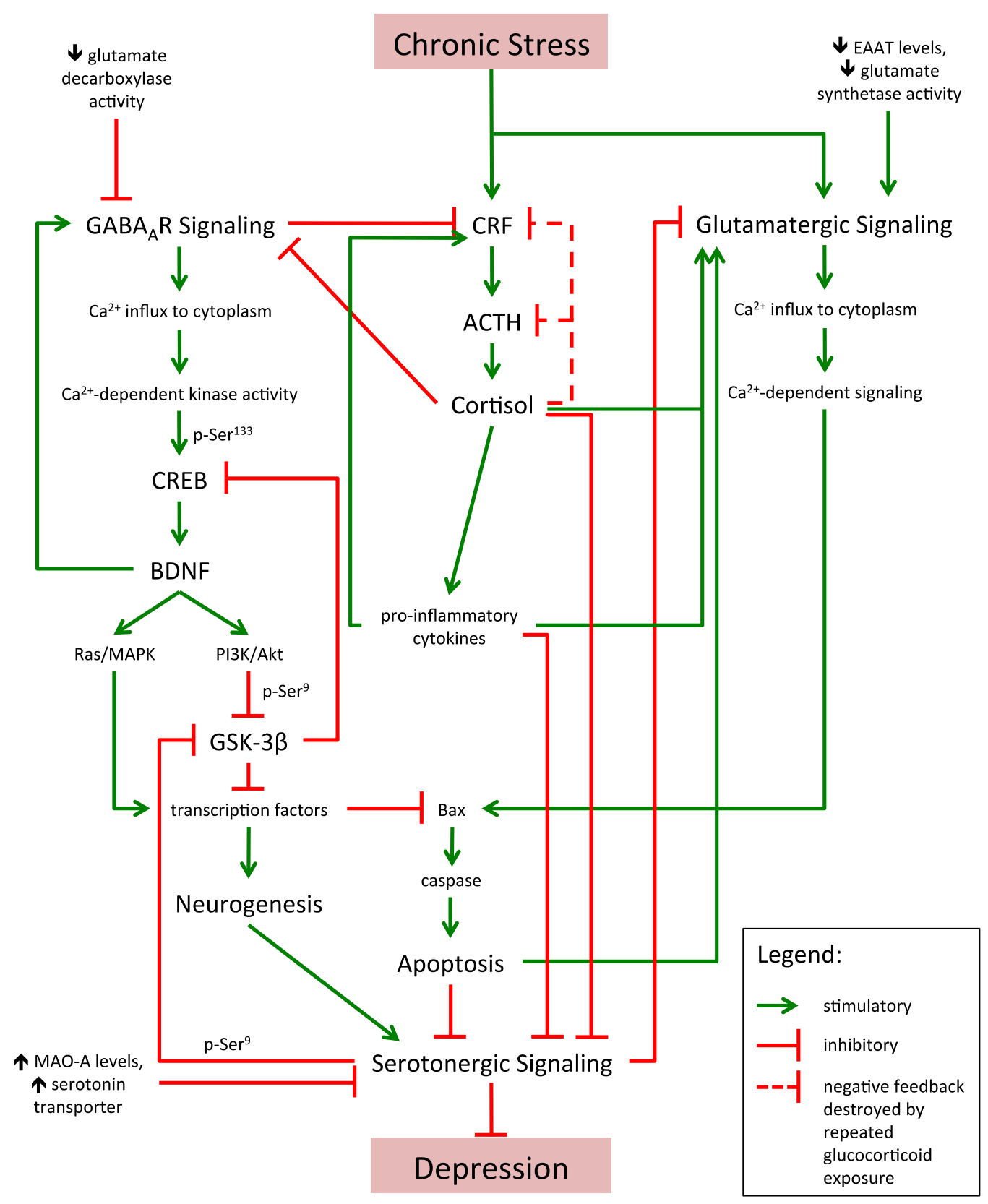

Figure 1. The etiology of depression according to Western medicine theory. GABA, $\gamma$-aminobutyric acid; GABAAR, GABAA receptor; CREB, cAMP response element-binding protein; BDNF, brain-derived neurotrophic factor; MAPK, mitogen-activated protein kinase; PI3K, phosphoinositide 3-kinase; GSK-3 $\beta$, glycogen synthase kinase $3 \beta$; p-Ser133, phosphorylation at Serine-133; CRF, corticotropin-releasing factor; ACTH, adrenocorticotropic hormone; EAAT, excitatory amino acid transporter.

neurons [7]. Monoamine oxidase inhibitors (MAOIs) interfere with neurotransmitter degradation following their reuptake into presynaptic terminals [10].

While antidepressant medications in Western medicine have been proven to be clinically effective, their use has been limited by potentially serious side effects. For example, MAOIs interact with certain foods or medications, causing high fever, disseminated intravascular coagulation, muscle rigidity, convulsions, or even coma [11]. Increased suicidal ideation is another possible side effect of antidepressant medications [12]. Moreover, currently used antidepressants provide only temporary relief of symptoms but are not curative. Symptoms of de- 
pression can return if the patient stops the medication [13]. Therefore, it is of great therapeutic interest to explore alternative treatments with fewer side effects and improved therapeutic efficacy.

Traditional Chinese medicine (TCM) is a time-honored practice in China and many Asian countries. While Western medicine adopts a reductionist approach to elucidating the biochemical basis of diseases, TCM views body function holistically on the basis of more abstract theories. A practitioner of Chinese medicine treats illness by first identifying the underlying cause or "syndrome" [14]. In this regard, TCM treatment can be curative rather than merely symptom-relieving. Furthermore, TCM makes use of multiple ingredients in herbal prescriptions to synergize therapeutic actions while minimizing toxicity to the body [15]. The characteristic features of TCM may confer advantages over the Western medicine approach to treating depression.

\section{Pathogenesis of Depression in TCM}

Depression is called yi-yu-zheng in Chinese. Transliterally, yi-yu means "clogged" or "stagnant" status of qi. In TCM, yu describes a collection of syndromes that are characterized by the obstruction of qi circulation [16]. Many disorders including depression are attributable to qi stagnation, of which the treatment involves the prescription of herbs that promote qi decongestion. Generally referred to as "vital energy" in the Western world, qi can be viewed as the functional manifestation of organs in the body [11] [16]. Qi can also be regarded as a substance partially derived from dietary nutrients, which nourishes every organ in the body [11]. Qi can be manifested in visible forms such as blood, mucous and serous fluids. In addition, qi can favor the conversion of food into fuel molecules, which then undergo oxidative phosphorylation to generate ATP. In this regard, ATP can be viewed as the biochemical unit of qi in the cell. While qi cannot be measured directly, problems related to qi can manifest as physiological disorders [16].

The liver plays an indispensable role in qi circulation. Through regulating the flow and activity of qi, the liver promotes optimal circulation of blood as well as digestion and metabolism in the spleen and stomach [16]. Liver synthesizes plasma proteins, such as albumin, which maintain the osmotic pressure of the blood [11]. Without these proteins, water would leave blood vessels through osmosis, causing edema in tissues and hypovolemia in the blood. Furthermore, the liver metabolizes and transforms carbohydrates, lipids, and amino acids for dissemination through the circulatory system. Hence, the proper flow and function of liver qi is crucial to one's health. Indeed, the importance of liver qi is reflected in the practice of TCM which uses various means for "soothing the liver" to relieve stress and anxiety [16].

Two factors can contribute to the aggravation of liver qi stagnation: phlegm and anger. Qi interacts intimately with blood, mucous fluid and serous fluid. When qi becomes clogged, other fluids also lose the driving force allowing them flowing smoothly [11]. Dampness then accumulates and eventually transforms into phlegm, which is sticky and can further inhibit the flow of qi [16]. If phlegm invades body regions above the chest, it can cause mental confusion. On the other hand, the "seven emotions" in TCM, namely, pleasure, anger, anxiety, grief, fear, shock, and melancholy, are also related to the functioning of specific organs. Anger can impair liver function, and when it acts in concert with stress, the optimal circulation of liver qi will be impaired.

According to the "five-element" theory, wood (the liver) generates fire (the heart or brain), which is indicative of the supportive role of liver in brain function. Liver qi stagnation can therefore manifest itself in the malfunctioning of the central nervous system (Figure 2). For instance, the obstruction of bile flow causes an increase in bilirubin concentration in the liver and then in the blood, resulting in jaundice with yellow pigment visible in the eyes [11]. Furthermore, the increased amount of bilirubin can enter the brain through an opened blood-brain barrier, where it is differentially accumulated in the hippocampus and basal ganglia, with resultant neurotoxicity [17]. As newborn babies have an underdeveloped and hence highly permeable blood-brain barrier, they are especially vulnerable to this type of neurotoxicity.

Given the current understanding of depression in both Western medicine and TCM perspectives, one can hypothesize that the blockage in liver qi influences the endocrine and central nervous systems, thereby causing depression. In the following section, we will examine these processes in further detail and suggest biochemical mechanisms whereby Chinese herbs might exhibit their antidepressant properties.

\section{Anti-Depressant Effects of TCM Formulations and Chinese Herbs}

Pharmacological studies have been conducted on TCM formulations which contain a typical liver-soothing herb, Bupleuri Radix (Bupleurum chinense) (Chaihu in Chinese or Saiko in Japanese), as their main herbal component. 

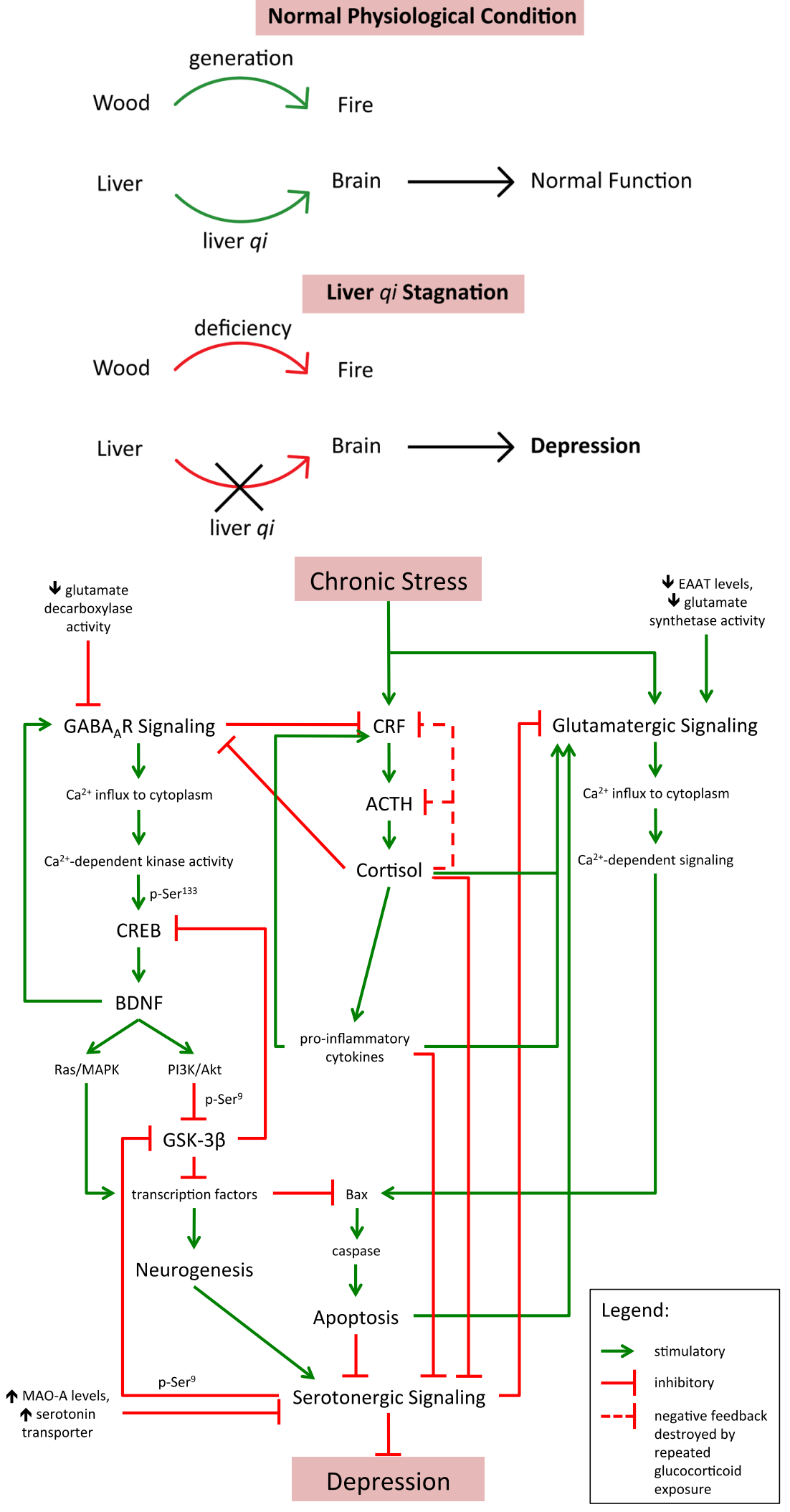

Figure 2. A schematic diagram of liver qi circulation between the liver and the brain under normal physiological conditions and depression. 
The most representative prescription is Chaihu-Shugan-San, which contains seven constituent herbs: Bupleuri Radix, Aurantii Nobilis Pericarpium (Citrus reticulata), Cnidii Rhizoma (Ligusticum striatum), Cyperi Rhizoma (Cyperus rotundus), Paeoniae Alba Radix (Paeonia lactiflora), Aurantii Fructus (Poncirus trifoliata) and Glycyrrhizae Radix (Glycyrrhiza uralensis) [18]. Other popular TCM formulations include Saiko-Ka-Ryukotsu-Borei-To, which containseleven constituent herbs: Bupleuri Radix, Ginseng Radix (Panax ginseng), Pinelliae Rhizoma (Pinellia ternata), Poria Cocos (Wolfiporia extensa), Scutellariae Radix (Scutellaria baicalensis), Cinnamomi Cortex (Cinnamomum cassia, Cinnamomum verum), Rhei Radix et Rhizoma (Rheum palmatum), Concha Ostreae (Ostrea gigas), Os Draconis (noted that Os Draconis is a fossil), Zingiberis Recens Rhizoma (Zingiber officinale) and Ziziphi Jujubae Fructus (Ziziphus jujuba) [19]. Chronically stressed rats have been the most commonly used animal model for depression. In brief, animals were orally administered TCM decoctions or their individual constituents for assessing their effectiveness in ameliorating depressive behavior. Studies found that Chaihu-Shugan-San and Bupleuri Radixcan ameliorate depression in a rat model of depression, as evidenced by an increase in sucrose consumption and a decreased immobility in the forced swimming test [17] [19] [20]. Saiko-Ka-Ryukotsu-Borei-To also increased riding time in a rotarod test in chronically stressed rats. Mizoguchiet al found that Saiko-Ka-Ryukotsu-Borei-To increased extracellular concentrations of monoamine neurotransmitters in the prefrontal cortex of chronically stressed rats [21]. The stimulatory effect on monoamines produced by the herbal preparation was found to be associated with a restoration of negative feedback regulation of the HPA axis [22].

\subsection{Bupleuri Radix Up-Regulates Downstream Signaling in the GABA Pathway}

A study using cultured neurons examined the effects of Bupleuri Radix on four downstream regulators in the GABA pathway, namely, CREB, BDNF, Akt, and GSK-3 $\beta$. It was found that the herb produced significant effects on these four signaling proteins in a dose-dependent manner. Bupleuri Radix promoted CREB phosphorylation, which was associated with corresponding increases in BDNF expression. Bupleuri Radix also promoted Akt phosphorylation. By activating the PI3K/Akt pathway, Bupleuri Radix up-regulated the phosphorylation and thus the inhibition of GSK-3 $\beta$, thereby protecting downstream transcription factors against GSK-3 $\beta$-mediated inactivation. These transcription factors could then promote neuronal cell survival, differentiation, and proliferation [19].

\subsection{Chaihu-Shugan-San Increases ERK5 Phosphorylation}

Extracellular-signal-regulated kinase 5 (ERK5) is a mitogen-activated protein kinase that, when phosphorylated, it promotes neurogenesis through the Ras/MAPK signaling pathway. In a study using a rat model, ERK5 expression in the hippocampus of chronically depressed rats was found to be similar to that of control rats, but the expression of p-ERK5 was much lower. The administration of Chaihu-Shugan-San up-regulated ERK5 phosphorylation in depressed rats, with the restoration of p-ERK5 levels to normal values. Furthermore, the constituent herbs in Chaihu-Shugan-San were divided into two groups for testing: Bupleuri Radix, Aurantii Nobilis Pericarpium, Cyperi Rhizoma, and Aurantii Fructus comprised Component I; while Cnidii Rhizoma, Paeoniae Alba Radix, and Glycyrrhizae Radix constituted Component II. The effect of Chaihu-Shugan-San in promoting ERK5 phosphorylation was much more significant than that of either Component I or II alone [21].

\subsection{Saiko-Ka-Ryukotsu-Borei-To Protects Neurons from Glutamate Excitotoxicity in the Hippocampus}

A study of Saiko-Ka-Ryukotsu-Borei-To utilizing zinc-deficient rats as a model for disrupted glutamatergic and GABAergic signaling showed that stimulation of hippocampal neurons with $\mathrm{K}^{+}$caused a marked increase in the release of glutamate but no detectable release of GABA. Treatment with Saiko-Ka-Ryukotsu-Borei-To completely suppressed the increase in extracellular glutamate concentrations in control rats. In zinc-deficient rats, the decoction also significantly suppressed the increase in glutamate release [23]. These results demonstrated that Saiko-Ka-Ryukotsu-Borei-To can prevent excessive glutamate release, thereby protecting the hippocampus against excitotoxicity, which is the death or damage of neurons caused by high levels of excitatory neurotransmitters such as glutamate.

In addition, the effect of Saiko-Ka-Ryukotsu-Borei-To on $\mathrm{K}^{+}$-induced GABA release in the hippocampus was 
also examined. While zinc-deficient rats showed no increase in extracellular GABA concentration following stimulation, Saiko-Ka-Ryukotsu-Borei-To administration significantly restored the increase in GABA release [23]. Hence, the decoction could rectify defective GABAergic neurotransmission by promoting GABA release.

\subsection{Saiko-Ka-Ryukotsu-Borei-To Inhibits the Intrinsic Apoptotic Pathway}

Chronic stress promotes apoptosis in hippocampal neurons. In the intrinsic apoptotic pathway, the mitochondrial inner membrane becomes permeable and releases cytochrome c into the intermembrane space and then into the cytoplasm. Cytochrome c recruits other cytoplasmic proteins to form an apoptosome, which subsequently induces a caspase cascade that leads to apoptosis [24]. In a study using a mouse model of depression, animals treated with Saiko-Ka-Ryukotsu-Borei-To had a significantly lower apoptotic index in the hippocampus when compared to depressed mice that were untreated. It was found that the administration of Saiko-Ka-RyukotsuBorei-To significantly down-regulated the expression of the apoptotic regulators Bax and caspase-3, thereby preventing cell death [19].

\section{Future Trends in Research on Antidepressant TCM Formulations and Chinese Herbs}

As mentioned in an earlier section, most pharmacological studies of TCM formulations have used a decoction of a complete herbal preparation. As far as the anti-depressant activity is concerned, studies using fractions of a decoction are rare, and it is even more difficult to find studies on individual herbal components in a given formulation. The investigation of each component herb is instrumental for identifying the active ingredient(s) and elucidating the mechanism(s) underlying their pharmacological actions. The study of Bupleuri Radix extract by Seo et al. provides an example of how this can be done [20].

In addition, TCM formulations are known to contain herbs that interact synergistically to produce their therapeutic effect, reduce toxicities of other component herbs, and enhance the bioavailability of the active ingredient(s) [15]. Future studies should focus on defining the modes of action of TCM formulations with antidepressant activity. To investigate synergistic interactions among herbal components, a rodent model of depression can be developed to assess behavioral parameters. Results obtained from these studies could form the basis for optimizing the herbal formulation used for treating depression.

\section{Pathological Linkage between Liver Qi Stagnation and Depression}

TCM views the liver as a crucial organ in the regulation of qi. Firstly, the liver promotes the generation of $q i$, wherein it metabolizes carbohydrates, lipids, and amino acids from foodstuffs into fuel molecules [16]. Secondly, the liver promotes the dissemination of these fuel molecules through the blood by synthesizing plasma proteins that maintain blood volume and thus a proper blood flow [11]. Individual cells can then take up the fuel molecules and convert them into ATP through oxidative metabolism. In the cell, ATP acts as the biochemical unit of $q i$, which is universally required for cellular activities.

ATP plays a particularly important role in the functioning of the nervous system. Indeed, ATP hydrolysis is a crucial step in the accumulation of neurotransmitters inside synaptic vesicles. The vesicle-associated enzyme, vacuolar- $\mathrm{H}^{+}$ATPase (V-ATPase), has two domains: V0 and V1. In the axon terminal, the catalytic cytoplasmic domain (V1) hydrolyzes ATP, thereby driving the transport of protons into the synaptic vesicle. Neurotransmitter-proton antiporters then make use of the resultant electrochemical proton gradient to fill the synaptic vesicle with a specific neurotransmitter. Hence, the hydrolysis of ATP (i.e., biochemical unit of qi) drives the process of neurotransmission [25].

Furthermore, ATP hydrolysis facilitates the release of neurotransmitters into the synaptic cleft. According to the "SNARE hypothesis" of synaptic transmission, neurotransmitters are released in two steps: vesicle docking and membrane fusion. Vesicle docking occurs when the SNARE proteins on the vesicle and the cytoplasmic membrane interact with each other to form a helical complex. When the neuron is stimulated and calcium ions are sequestered in the axon terminal, the N-ethylmaleimide-sensitive fusion protein (NSF) dissociates the SNARE complex and induces membrane fusion, resulting in the release of neurotransmitters into the synaptic cleft. The SNARE complex is extremely stable and the hydrolysis of ATP is required for its disassembly. Therefore, NSF functions as an ATPase that drives the release of neurotransmitters at the expense of ATP [26]. 
In addition, ATP is indispensable in the trafficking of growth signals. Neurotrophins first enter the target neuron at the axon terminal through receptor-mediated endocytosis. They subsequently undergo retrograde transport to the nucleus and activate genes that promote cell growth and survival. The process of retrograde transport requires the motor protein dynein, which carries the endosome vesicle along the microtubule. ATP hydrolysis produces conformational changes in dynein, which enables it to be transported along the length of the neuron. Therefore, dynein consumes ATP to energize the transport of growth factors in the neuron. Without a sufficient supply of ATP, neurons do not receive adequate growth signals and neurodegeneration occurs [27], leading to defective neurotransmission.

\section{Conclusion}

Recent studies in Western medicine have shown that depression is likely caused by the dysregulation of neurotransmission, which, according to TCM theory, is a consequence of liver $q$ i stagnation. It is suggested that the experience of anger and/or stress disrupts qi circulation in the liver, leading to a deficiency in fuel molecule production and plasma protein synthesis. Thus, the hippocampus and prefrontal cortex are not sufficiently nourished by $q i$, leading to defective neurotransmitter signaling resulting in depression. Based on this understanding, the etiology of depression in terms of Chinese medicine theory becomes self-explanatory, and it serves as a rationale for the treatment of depression using herbal formulations.

\section{References}

[1] World Health Organization (2012) Depression Fact Sheet. http://www.who.int/mediacentre/factsheets/fs369/en/

[2] Lee, S., Tsang, A. and Kwok, K. (2007) Twelve-Month Prevalence, Correlates, and Treatment Preference of Adults with DSM-IV Major Depressive Episode in Hong Kong. Journal of Affective Disorders, 98, 129-136. http://dx.doi.org/10.1016/j.jad.2006.07.009

[3] National Collaborating Centre for Mental Health (UK) (2010) Depression: The Treatment and Management of Depression in Adults. National Institute for Health and Clinical Excellence: Guidance, The British Psychological Society \& The Royal College of Psychiatrists, Leicester.

[4] Bayat, N., Alishiri, G.H., Salimzadeh, A., Izadi, M., Saleh, D.K., Lankarani, M.M. and Assari, S. (2011) Symptoms of anxiety and Depression: A Comparison among Patients with Different Chronic Conditions. Journal of Research in Medical Sciences, 16, 1441-1447.

[5] Hasler, G. (2010) Pathophysiology of Depression: Do We Have Any Solid Evidence of Interest to Clinicians? World Psychiatry, 9, 155-161. http://dx.doi.org/10.1002/j.2051-5545.2010.tb00298.x

[6] Holsboer, F. and Ising, M. (2010) Stress Hormone Regulation: Biological Role and Translation into Therapy. Annual Review of Psychology, 61, 81-109. http://dx.doi.org/10.1146/annurev.psych.093008.100321

[7] Werner, F.M. and Coveñas, R. (2010) Classical Neurotransmitters and Neuropeptides Involved in Major Depression: A Review. International Journal of Neuroscience, 120, 455-470. http://dx.doi.org/10.3109/00207454.2010.483651

[8] Leonard, B.E. (2010) The Concept of Depression as a Dysfunction of the Immune System. Current Immunology Reviews, 6, 205-212. http://dx.doi.org/10.2174/157339510791823835

[9] Zunszain, P.A., Anacker, C., Cattaneo, A., Carvalho, L.A. and Pariante, C.M. (2011) Glucocorticoids, Cytokines and Brain Abnormalities in Depression. Progress in Neuro-Psychopharmacology \& Biological Psychiatry, 35, $722-729$. http://dx.doi.org/10.1016/j.pnpbp.2010.04.011

[10] Medscape (2014) Monoamine Oxidase Inhibitor Toxicity. http://emedicine.medscape.com/article/815695-overview

[11] Kong, L.D., Cheng, C.H.K. and Tan, R.X. (2001) Monoamine Oxidase Inhibitors from Rhizoma of Coptis chinensis. Planta Medica, 67, 75-76. http://dx.doi.org/10.1055/s-2001-10874

[12] Reeves, R.R. and Ladner, M.E. (2010) Antidepressant-Induced Suicidality: An Update. CNS Neuroscience \& Therapeutics, 16, 227-234. http://dx.doi.org/10.1111/j.1755-5949.2010.00160.x

[13] DuRubeis, R.J., Siegle, G.J. and Hollon, S.D. (2008) Cognitive Therapy versus Medication for Depression: Treatment Outcomes and Neural Mechanisms. Nature Reviews Neuroscience, 9, 788-796. http://dx.doi.org/10.1038/nrn2345

[14] Kong, Y.C. (2005) The Cultural Fabric of Chinese Medicine: How to Know Your Body through Chinese Medicine. The Commercial Press, Hong Kong.

[15] Tsim, K.W.K. (2015) The Genesis of Traditional Chinese Medicine, “The Divine Farmer’s Herb-Root Classic”. http://www.master-insight.com/content/article/2911

[16] Hong, H. (2014) The Theory of Chinese Medicine: A Modern Interpretation. Imperial College Press, London. 
http://dx.doi.org/10.1142/p933

[17] Levine, R.L., Fredericks, W.R. and Rapoport, S.I. (1982) Entry of Bilirubin into the Brain Due to Opening of the Blood-Brain Barrier. Pediatrics, 69, 255-259.

[18] Kim, S.H., Han, J., Seog, D.H., Chung, J.Y., Kim, N., Park, Y.H. and Lee, S.K. (2005) Antidepressant Effect of Chaihu-Shugan-San Extract and Its Constituents in Rat Models of Depression. Life Sciences, 76, 1297-1306. http://dx.doi.org/10.1016/j.lfs.2004.10.022

[19] Liu, Y., Ma, S. and Qu, R. (2010) SCLM, Total Saponins Extracted from Chaihu-jia-longgu-muli-tang, Reduced Chronic Mild Stress-Induced Apoptosis in the Hippocampus in Mice. Pharmaceutical Biology, 48, 840-848. http://dx.doi.org/10.3109/13880200903296154

[20] Qiu, J., Hu, S.Y., Zhang, C.H., Shi, G.Q., Wang, S.E. and Xiang, T. (2014) The Effect of Chaihu-Shugan-San and Its Components on the Expression of ERK5 in the Hippocampus of Depressed Rats. Journal of Ethnopharmacology, 152, 320-326. http://dx.doi.org/10.1016/j.jep.2014.01.004

[21] Mizoguchi, K., Yuzurihara, M., Ishige, A., Aburada, M. and Tabira, T. (2003) Saiko-ka-ryukotsu-borei-to, a Herbal Medicine, Ameliorates Chronic Stress-Induced Depressive State in Rotarod Performance. Pharmacology Biochemistry \& Behavior, 75, 419-425. http://dx.doi.org/10.1016/s0091-3057(03)00131-X

[22] Mizoguchi, K., Yuzurihara, M., Ishige, A., Sasaki, H. and Tabira, T. (2002) Saiko-ka-ryukotsu-borei-to, a Herbal Medicine, Prevents Chronic Stress-Induced Disruption of Glucocorticoid Negative Feedback in Rats. Life Sciences, 72, 67-77. http://dx.doi.org/10.1016/S0024-3205(02)02199-9

[23] Tamano, H. and Takeda, A. (2004) Suppressive Effect of Saiko-ka-ryukotsu-borei-to, a Herbal Medicine, on Excessive Release of Glutamate in the Hippocampus. Brain Research Bulletin, 64, $273-277$. http://dx.doi.org/10.1016/j.brainresbull.2004.07.013

[24] Martinou, J.C. and Youle, R.J. (2011) Mitochondria in Apoptosis: Bcl-2 Family Members and Mitochondrial Dynamics. Developmental Cell, 21, 92-101. http://dx.doi.org/10.1016/j.devcel.2011.06.017

[25] Morel, N. (2003) Neurotransmitter Release: The Dark Side of the Vacuolar- $\mathrm{H}^{+}$ATPase. Biology of the Cell, 95, 453457. http://dx.doi.org/10.1016/S0248-4900(03)00075-3

[26] Chen, Y.A. and Scheller, R.H. (2001) SNARE-Mediated Membrane Fusion. Nature Reviews Molecular Cell Biology, 2, 98-106. http://dx.doi.org/10.1038/35052017

[27] Cosker, K.E., Courchesne, S.L. and Segal, R.A. (2008) Action in the Axon: Generation and Transport of Signaling Endosomes. Current Opinion in Neurobiology, 18, 270-275. http://dx.doi.org/10.1016/j.conb.2008.08.005 